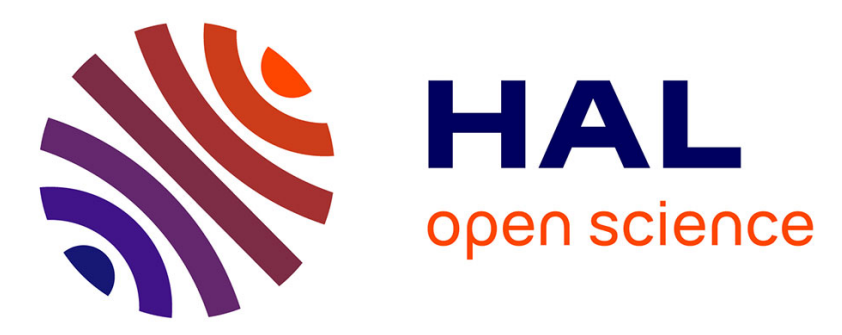

\title{
La voie étroite de la doctrine française aux États-Unis : étude des références faites aux juristes français dans les law reviews duTexas,de la Californie et du Michigan
} Prune Decoux

\section{- To cite this version:}

Prune Decoux. La voie étroite de la doctrine française aux États-Unis : étude des références faites aux juristes français dans les law reviews duTexas,de la Californie et du Michigan. Clio@Thémis. Revue électronique d'histoire du droit, 2018. hal-02943017

\section{HAL Id: hal-02943017 \\ https://hal.science/hal-02943017}

Submitted on 25 Feb 2021

HAL is a multi-disciplinary open access archive for the deposit and dissemination of scientific research documents, whether they are published or not. The documents may come from teaching and research institutions in France or abroad, or from public or private research centers.
L'archive ouverte pluridisciplinaire HAL, est destinée au dépôt et à la diffusion de documents scientifiques de niveau recherche, publiés ou non, émanant des établissements d'enseignement et de recherche français ou étrangers, des laboratoires publics ou privés. 


\title{
La voie étroite de la doctrine française aux États-Unis ${ }^{1}$ : étude des références faites aux juristes français dans les law reviews du Texas, de la Californie et du Michigan.
}

\begin{abstract}
Résumé : Entre 1900 et 1950, les revues juridiques universitaires des États-Unis font de nombreuses références à la doctrine française, par le biais de comptes rendus d'ouvrage, de traductions ou de commentaires d'articles. Dès lors, le but de cette étude est d'étudier la circulation des idées entre ces deux continents : par exemple, cette diffusion de la pensée française se fait-elle de façon uniforme sur le territoire ou correspond-elle à des pratiques étatiques? Cette diffusion est-elle réelle ou se voit-elle confinée à quelques universitaires francophiles ? Pour tenter d'éclairer le régime circulatoire en vigueur, nous concentrerons notre attention sur les laws reviews de trois États, le Texas, le Michigan et la Californie, mettant en œuvre la méthode structurale afin de cartographier les réseaux existants et de classer les références trouvées.
\end{abstract}

\begin{abstract}
Many cross-links were established between American and French scholars. They were intertwined and they related to each other: they sent them their books, they commented or interpreted their works. In this way, there were numerous references to the French Legal though in the laws reviews. These intellectual connections raise questions: how effective are the traditional circulatory systems? Is there an American way of thinking, an American way of assimilating, or rather the opposite is there specificity by states in the reception and assimilation of these ideas? Is there a real spreading of the French legal thought across the continent, or is it still primarily confined to some Francophile scholars? We hope to bring some food for thought through a systematic and statistical and qualitative survey about the law reviews of the Michigan, Texas, and California throughout the first half of the twentieth century.
\end{abstract}

Mots-clés : circulation des idées - Histoire de la pensée juridique - Analyse de réseaux France et États-Unis

Key-words : Circulation of Ideas - History of Legal Thought - Structural Analysis - France and United States

1. " "Incertitude" est l'un des mots qui, par bien des aspects, permet de qualifier l'historiographie actuelle $»^{2}$. Si ce constat est formulé à propos de la matière historique à proprement parler, jugée à la fin $\mathrm{du} \mathrm{XX}^{\mathrm{e}}$ siècle trop abstraite, trop déconnectée et trop désireuse de structures au détriment des récits ${ }^{3}$, il nous semble pouvoir assez bien s'appliquer à l'historiographie en matière d'histoire du droit. Longtemps vue, voire autoproclamée, comme neutre idéologiquement, politiquement et sociologiquement ${ }^{4}$, l'histoire du droit semble désormais subir un changement de perspective en usant notamment de "méthodes

\footnotetext{
${ }^{1}$ Ce titre en forme de clin d'œil renvoie au texte célèbre d'Oliver Wendell Holmes, "The Path of the Law », Harvard Law Review, 10, 1897, p. 457-478, traduit par Françoise Michaut, "La passe étroite du droit», Clio@Thémis, 2, 2009, [en ligne].

${ }^{2}$ J.-Y. Grenier, « Temporalités, incertitude et historiographie », Communications, 95, 2014, p. 119.

${ }^{3}$ Ibidem, p. 120.

${ }^{4}$ F. Audren, «Introduction : 1'histoire intellectuelle du droit ou la fin du "Grand Partage" », Clio@Thémis, 9, 2015 [en ligne].
} 
ressortissant de l'histoire sociale, culturelle ou intellectuelle $»^{5}$. Il reste peut-être quelques écueils, tels que le fait que ce soient les juristes qui produisent leurs propres manuels d'histoire générale, à destination de leurs propres étudiants : cette «autonomie persistante d'une histoire des juristes $\rangle^{6}$ peut d'ailleurs présenter quelques points de ressemblances avec l'histoire internaliste en vogue au XIX ${ }^{\mathrm{e}}$ siècle aux États-Unis ${ }^{7}$.

2. Toujours est-il que l'heure semble être à la remise en question et surtout à la remise en situation. De plus, auparavant rendue obsolète par une approche macro de l'histoire, la microhistoire semble revenir sur le devant de la scène, portant son intérêt sur l'individu, l'individu dans son entièreté «afin de rester au plus près du sens porté par le document » ${ }^{8}$. Cette recherche de sens, ou cette tentative de ne pas trop s'écarter du sens porté par le texte, nous semble imposer une troisième réflexion: après la situation et l'individuation, il semble important d'examiner la narration du texte. L'analyse du discours et les phénomènes de transtextualité paraissent susceptibles d'apporter un autre degré de compréhension, ou simplement des éclaircissements... ou encore plus modestement une réduction de la marge d'incertitudes.

3. Face à ces nouvelles demandes, l'usage de nouveaux outils ${ }^{9}$ paraît offrir des possibilités intéressantes. Produire une histoire "connectée » à son époque apparait facilité dans notre époque elle-même si connectée : dans les année 1970, Emmanuel Le Roy Ladurie prophétisait que « grâce à l'ordinateur, l'historien devenait un scientifique » ${ }^{10}$. Le rapprochement implicite d'avec les sciences improprement dites « exactes» ou «dures" peut prêter à sourire ou à grincer des dents ; dans tous les cas, il semble difficile, encore plus de nos jours qu'à l'époque de l'approche quantitative, d'échapper à «l'idée que le chiffre, par sa rigueur et sa précision, apporte une scientificité supérieure qui réduit l'incertitude de la connaissance historique $»^{11}$. Sans agréer ni démentir, il nous semble que le chiffre ou les statistiques peuvent en effet être utilisés à bon escient dans la recherche historique, comme le démontrent les humanités numériques. Bases de données, data mining, analyse de réseaux.... nous paraissent autant d'importations possibles susceptibles de « renouveler la compréhension de ces phénomènes à partir d'un détour hors du champ juridique $\rangle^{12}$, et par là même, autant de possibilités de renouveler l'historiographie de l'histoire du droit.

4. Ces outils spécifiques ${ }^{13}$ ont été utilisés dans cette étude en vue d'étudier la présence de la doctrine juridique française dans le champ étatsunien ${ }^{14}$. Cette présence semble bien réelle : les universitaires étasuniens et français interagissent entre eux, s'envoient des ouvrages, commentent ou interprètent leurs travaux respectifs, s'invitent dans leurs facultés respectives,

\footnotetext{
${ }^{5}$ L. Guignard et G. Malandain, «Introduction : usages du droit dans l'historiographie du XIX ${ }^{\mathrm{e}}$ siècle », Revue d'histoire du XIX siècle, 48, 2014/1, p. 16.

${ }^{6}$ Ibidem, p. 6.

${ }^{7}$ D. Rabban, « The Historiography of Late Nineteenth-Century American Legal History », Theoretical Inquiries in Law, 4/2, juillet 2003, p. 541-578. Voir ce numéro.

${ }^{8}$ J.-Y. Grenier, « Temporalités, incertitude et historiographie », art. cit., p. 125.

${ }^{9}$ Le terme « nouveau » est à entendre ici comme « nouveau dans la recherche juridique »; l'analyse de réseaux est par exemple utilisée depuis très longtemps en sociologie.

${ }^{10}$ J.-Y. Grenier, « Temporalités, incertitude et historiographie », art. cit., p. 119.

${ }^{11}$ Ibidem.

${ }^{12}$ F. Audren, « Introduction : l'histoire intellectuelle du droit ou la fin du “Grand Partage" », art. cit., [en ligne].

${ }^{13}$ Concernant la méthodologie employée, le lecteur se réfèrera utilement à l'article présent dans ce même dossier de N. Hakim et A. Monti, «Histoire de la pensée juridique et analyse bibliométrique : l'exemple de la circulation des idées entre la France et l'Italie à la Belle Époque » [LIEN en ligne], particulièrement aux $n^{\circ} 13$ et $\mathrm{s}$.

${ }^{14}$ Cette recherche a été présentée lors de la British Legal History Conference 2017 - Networks and Connections, 5-8 July 2017, UCL Faculty of law, London, England, organisée par Michael Lobban and Ian Williams.
} 
voire entretiennent des liens personnels. Reste toutefois à, si ce n'est quantifier, du moins étudier la densité de cette présence et son inscription ou non dans la description traditionnelle du modèle circulatoire des idées ${ }^{15}$. Dans ce cadre, il s'agit de modeler des réseaux intellectuels existant entre certaines universités américaines et des membres de la doctrine française, espérant ainsi pouvoir décrire le rôle joué par les citations d'auteurs français dans les revues juridiques américaines.

5. Les law reviews sont ainsi le support privilégié de ce travail : ce qui n'était à l'origine que des journaux étudiants destinés à proposer des extraits des cours professés est devenu au cours du temps un support doctrinal très important, réunissant les contributions des professeurs, avocats ou juges les plus célèbres de leur temps. Certaines d'entres elles ont ainsi joué un rôle décisif dans la diffusion et l'unification de la pensée juridique sur le continent étatsunien ${ }^{16}$. Parmi elles, quatre ont été étudiées : la California Law Review, la South California Law Review, la Michigan Law Review et la Texas Law Review. Ce choix est motivé par différentes raisons, telles que l'éviction délibérée d' " universités-phares », faisant par exemple partie de la Ivy League. Il nous a en effet semblé que ces institutions, souvent anciennes et très prestigieuses, étaient plus à même d'attirer un public international, ce qui aurait pu se ressentir dans leurs publications. En dehors de ces considérations tendant à une harmonisation que nous savons bien utopique, des états différents, géographiquement et juridiquement, ont été intégrés; de plus, la Californie possède deux universités, et donc deux law reviews, spécifiquement choisies pour étudier les éventuelles différences entre institutions.

6. Initialement, le cadre temporel envisagé était celui de la $\mathrm{III}^{\mathrm{e}}$ République, où la révolution industrielle affecta profondément le tissu social et eut des répercussions sur la pensée juridique française comme étatsunienne. Pourtant, la période retenue a été plus étroitement délimitée, pour des raisons pragmatiques : la Michigan Law Review est la plus ancienne mais la review du Texas ne paraît qu'à partir de 1923. Ainsi, c'est la période 1923-1950 qui a été retenue afin de mettre ces revues sur un pied d'égalité quant à la fréquence des citations ou aux thèmes recensés.

7. Comme nous l'avons signifié, des outils particuliers ont été employés, demandant quelques éclairages méthodologiques. L'un de ces outils, largement employé dans le domaine de la sociologie, est la constitution et l'analyse de réseaux ${ }^{17}$. Construire un tel réseau nécessite de collecter en amont des données bibliométriques ${ }^{18}$. Ainsi, les law reviews précitées ont été dépouillées et chaque référence ou citation à un auteur de doctrine français a été recensée. Les références sont en effet particulièrement révélatrices de la circulation des idées et pour

\footnotetext{
15 P.-Y. Saunier, «Les régimes circulatoires du domaine social 1800-1940: projets et ingénierie de la convergence et de la différence », Genèses, 71, 2008/2, p. 4-25.

${ }^{16}$ E. N. Griswold, « The Harvard Law Review - Glimpses of Its History as Seen by an Aficionado », Harvard Law Review : Centennial Album I, 1987, p. 23 ; R. A. Posner, «Against the Law Reviews », Legal Affairs, Nov.Déc. 2004 [En ligne].

17 Voir par exemple T. Caplow, Deux contre un, Paris, A. Colin, 1984 ; M. Ferrary et Y. Pesqueux, L'organisation en réseau, mythes et réalités, Paris, Presses universitaires de France, 2004 ; E. Lazega, Réseaux sociaux et structures relationnelles, 3e éd., Paris, Presses universitaires de France, 2014 ; P. Mercklé, Sociologie des réseaux sociaux, $3^{\mathrm{e}}$ éd., Paris, La découverte, 2016.

${ }^{18}$ Concernant la bibliométrie et ses avantages, voir N. Hakim et A. Monti, « Histoire de la pensée juridique et analyse bibliométrique », art. cit., particulièrement les $\mathrm{n}^{\circ} 16$ et 18 et les $\mathrm{n}^{\circ} 21$ à 23 ; sur la collecte de données, Ibidem, $\mathrm{n}^{\circ} 26$; sur le logiciel utilisé pour visualiser de tels réseaux, Ibidem, n²7.
} 
certains auteurs, elles sont partie intégrantes de l'activité scientifique elle-même ${ }^{19}$. Ces citations sont ensuite représentées dans un réseau tel que le décrit Barnes :

Chaque personne est en contact avec un certain nombre de personnes, qui sont ellesmêmes en contact entre elles, ou non. [...] Je trouve cela pratique de décrire ce genre de champ social en terme de réseau. Je me le représente comme un ensemble de points [nœuds], certains étant reliés par des lignes [liens]. Les points de cette image sont les individus, ou parfois les institutions ; et les lignes indiquent quel individu interagit avec tel autre ${ }^{20}$.

Ici, les nœuds représenteront les law reviews ou les auteurs français et les liens les références trouvées.

8. En guise de vue d'ensemble, le réseau ci-dessous représente toutes les citations faites à des auteurs français par les quatre law reviews étudiées sur l'ensemble de la période envisagée. L'épaisseur des lignes, ou liens, est variable : plus l'auteur est mentionné, plus le lien est épais, ou «valué ». Seuls quelques noms ont été mis en exergue pour des raisons de lisibilité.

\section{Panorama général}

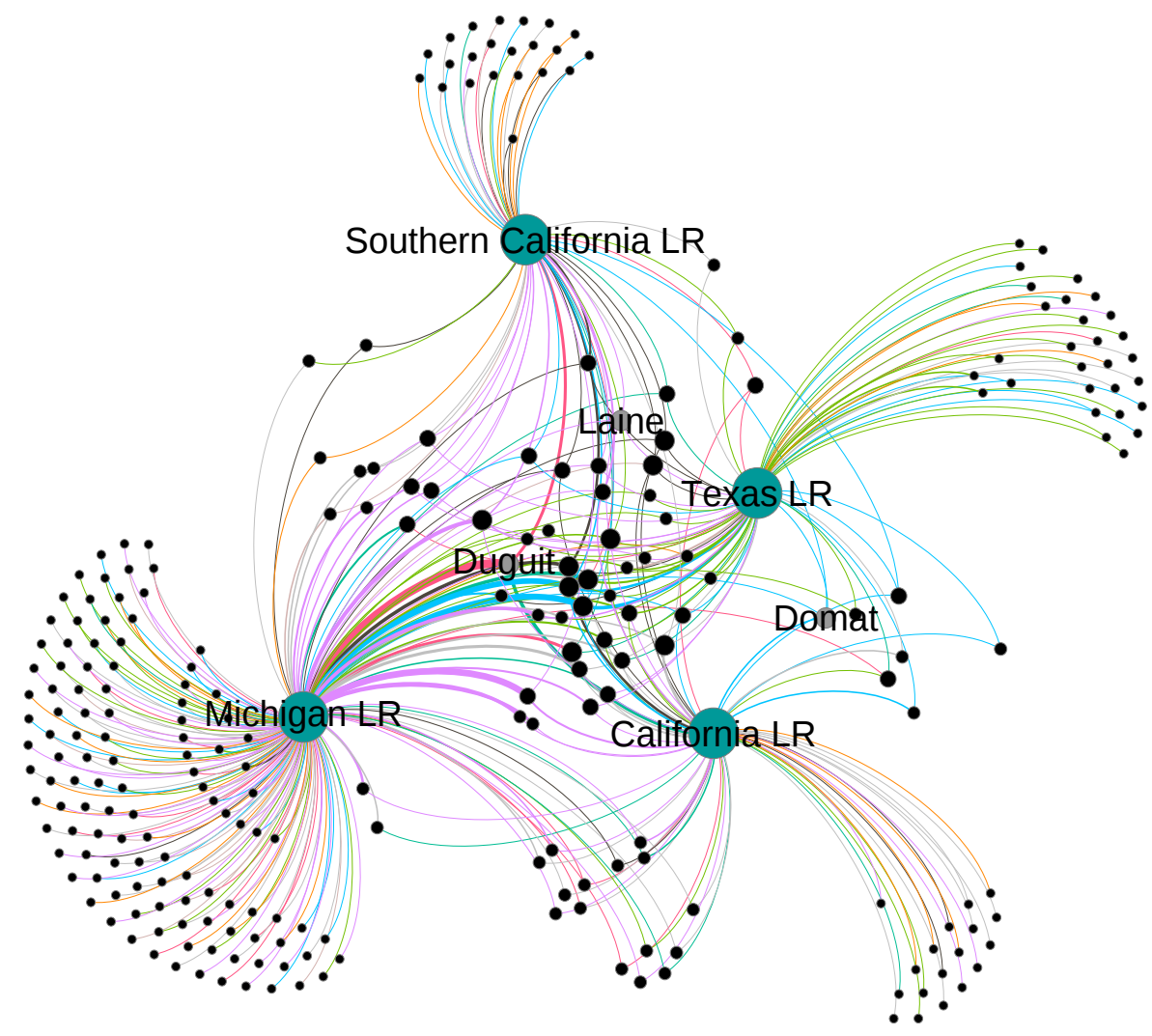

Représentations des références faites à la doctrine française entre 1870 et 1940 ; chaque couleur représente une discipline différente.

\footnotetext{
${ }^{19}$ B. Milard, "Quelles sociabilités derrière les références bibliographiques ? Citations et relations sociales », Socio-logos, 8, 2013 [En ligne]. Voir également N. Hakim et A. Monti, ibidem, n ${ }^{\circ} 15$ à 18 ainsi que la bibliographie abondante proposée à la note 12 du même article.

${ }^{20}$ J. A. Barnes, «Class and Committees in a Norwegian Island Parish », Human Relations, 71, 1954/1, p. 43.
} 
9. Avant d'aller plus loin dans l'analyse, ce premier réseau peut d'ores et déjà appeler quelques remarques. À première vue, le cœur du diagramme est très dense et enchevêtré, ce qui pourrait signifier une forte circulation des auteurs entre ces mêmes universités et/ou un champ scientifique partagé. Toutefois, on constate également que chacune de ces universités est accompagné de sa propre cohorte de références à des auteurs français, qu'elle ne partage pas avec une autre, qui se présente sous forme d'ombrelle ou de pissenlit ; celui du Michigan est sensiblement plus important que celui des autres, ce qui peut nous mettre sur la voie d'un état plus francophile ou plus fortement relié à la France que les autres.

10. Si cette première phase d'observation est nécessaire et peut parfois donner des orientations de recherche, il est absolument indispensable de pousser plus loin l'analyse afin d'infirmer ou de confirmer les hypothèses soulevées et en découvrir d'autres. Pour ce faire, nous partirons du graphique susdit en modulant différents facteurs. Ainsi, la variation des «degrés entrants ${ }^{21}$ nous permettra d'identifier les références partagées par certains professeurs de deux, trois ou quatre universités, et peut-être un intérêt spécifique pour un auteur en particulier ou son champ de compétence; cela sera contextualisé par des développements plus larges. De même, les différents sujets mobilisés par les universités seront étudiés, afin d'en observer les similarités et les dissemblances. Dès lors, les modulations selon le degré entrant seront suivies des modulations par sujets.

11. Dans les graphiques suivants, nous avons choisi de ne faire apparaître que certains auteurs, selon un critère précis, celui du degré entrant; en d'autres termes, le graphique montrant les réseaux de degré 1 fera apparaître la doctrine citée par une, et une seule université, tandis qu'un graphique de degré 2 ferait apparaître celle citée par deux universités, etc. Cette étude se concentrera volontairement sur les deux extrêmes, à savoir les réseaux de degré 1 et les réseaux de degré 4 .

\section{Les réseaux de degré 1}
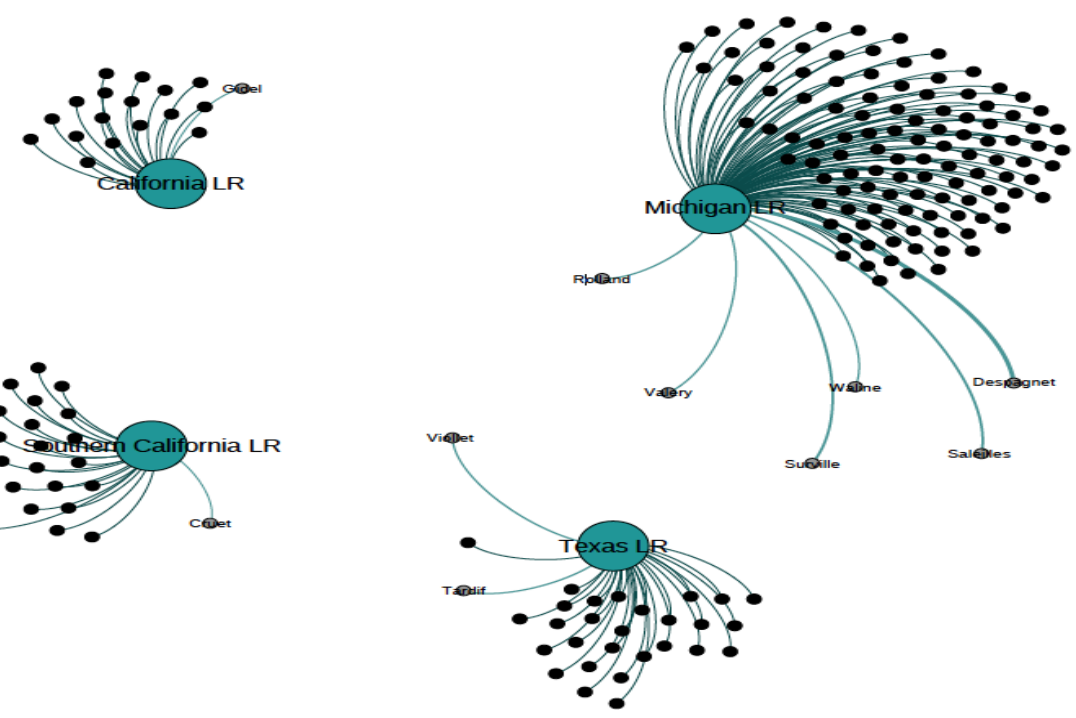

Cartographie des auteurs français cité une fois, et une seule, par une des universités représentées

\footnotetext{
${ }^{21}$ Le degré entrant est le lien nombre de liens vers $\mathrm{X}$, tandis que le degré sortant est le nombre de liens sortant de $\mathrm{X}$. Dans le diagramme ci-dessous, la California Law Review a un degré entrant de 0 (aucun auteur ne la cite) mais un degré sortant de 18 (elle cite dix-huit auteurs).
} 
12. Ce graphique peut apparaître comme de prime abord comme l'antithèse de l'analyse de réseaux, puisque le but est de faire apparaître les auteurs qui ne sont mentionnés que par une, et une seule université ; les entités apparaissent comme autant d'atomes solitaires sans lien entre eux. Pourtant, il est possible d'en tirer un premier constat intéressant, celui que chaque université possède son propre lot de références à la doctrine française. Or, une hypothèse aurait pu être faite, celui d'une utilisation "cliché » de la doctrine française, au même titre que les voilà ou c'est la vie utilisés par nos amis Outre-Atlantique : largement diffusée et partagée par tout le monde, à l'emploi un peu automatique et évoluant dans une sorte d'inconscient collectif. Or, ce type de diagramme ne va pas dans ce sens. Une étude quantitative des juristes mobilisés par ces différentes law reviews montrent que malgré des références non partagées, il existe des points communs entre universités dans la mobilisation des citations ; ces points communs côtoient toutefois des spécificités locales.

\section{1. Des points communs dans la mobilisation de la doctrine}

13. Ainsi, ces quatre universités présentent des similarités au vu des matières faisant intervenir des acteurs français. Mis à part le Texas, chacune de ces law reviews publie des articles de droit international, ce qui explique la présence d'auteurs tels que Despagnet, Surville, Gidel ou Potu ${ }^{22}$. Cet intérêt s'explique facilement : si certains auteurs considèrent le droit international comme « aussi vieux que la civilisation elle-même ${ }^{23}$, d'autres datent son avènement, du moins dans sa forme moderne, du Traité de Westphalie. L'extension du droit international a été un des faits majeurs de la seconde moitié du XIX ${ }^{\mathrm{e}}$ siècle, tandis que son universalisation a été l'enjeu du $\mathrm{XX}^{\mathrm{e}}$ siècle $^{24}$. La naissance des premières organisations internationales et les grands conflits mondiaux ont mis cette matière sur le devant de la scène, dans une société de plus en plus mondialisée : les communications se font plus rapides et les échanges s'intensifient, expliquant que de nombreux articles traitant de conflict of laws paraissent dans les supports juridiques étudiés ${ }^{25}$. Les auteurs français comportant quelques noms éminents en la matière, comme celui d'Antoine Pillet ${ }^{26}$, cela explique leur présence dans ce contexte.

14. De la même manière, ces universités se tournent également vers le droit comparé ${ }^{27}$, s'inscrivant dans le mouvement de pensée de leur époque : les premières années du $\mathrm{XX}^{\mathrm{e}}$ siècle

\footnotetext{
${ }^{22}$ Respectivement cités dans E. H. Ailes, "Limitation of Actions and the Conflict of Laws », Michigan Law Review, 31, 1933, p. 474-502 ; J. M. Cormack, «Renvoi, Characterization, Localization and Preliminary Question in the Conflict of Laws », Southern California Law Review, 14, 1941, p. 221-275 ; F. Deak, "The Rumanian-Hungarian Dispute before the Council of the League of Nations ", California Law Review, 16, 1928, p. $120-133$.

${ }^{23}$ S. A. Korff, «An Introduction to the History of International Law », American Journal of International Law, 18,1924, p. 246.

${ }^{24}$ R. Kolb, « Mondialisation et droit international », Relations internationales, 123, 2005/3, p. 69-86.

${ }^{25}$ Par exemple, A. D. McNair, «Equality in International Law », Michigan Law Review, 26, 1927, p. 131-152 ;

F. Deak, "Classification, Immunities and Privileges of Diplomatic Agents », Southern California Law Review, 1, 1928, p. 209-252 ; E. Borchard, "The "Minimum Standard" of the Treatment of Aliens », Michigan Law Review, 38, 1940, p. 445-461 ; J. M. Cormack, « Renvoi », art. cit. ; W. W. Jr. Bishop, « Conflict of Criminal Laws, de E. Stimson », California Law Review, 25, 1936-1937, p. 640.

${ }^{26} \mathrm{Cf}$. infra, $\mathrm{n}^{\circ} 32$.

27 H. S. Daggett, "The Chattel Mortgage in Louisiana», Texas Law Review, 16, 1938, p. 162-190 ; J. M. Cormack et F. F. Barker, "The Mexican Law of Business Organizations », Southern California Law Review, 6, 1932-1933, p. 181-228 ; F. F. Barker et J. M. Cormack, "The Mercantile Act : A Study in Mexican Legal Approach », Ibidem, 6, 1932, p. 1-30; B. Ramirez et M. Luis, "Capacity Under the Negotiable
} 
voient s'ouvrir une nouvelle ère pour le droit comparé aux États-Unis, du point de vue notamment de son organisation et surtout de son enseignement ${ }^{28}$. Nous ne trouvons pas dans ces articles les "grands noms » du droit comparé français, tels que Lambert, mais il ne faut pas oublier que nous sommes ici au stade du « degré 1 », c'est à dire de la référence unique à un auteur. Toutefois, il est impossible de ne pas remarquer la composante civiliste manifeste existant dans ces différents articles, puisque l'on y parle de la Louisiane, de l'Amérique latine ou du Mexique, ou encore du droit « continental ». Dès lors, les auteurs français ne semblent pas invoqués ici au titre de comparatistes, mais plus comme les spécialistes d'un point de droit, dérivé du système civiliste, visé par l'article ${ }^{29}$.

15. Outre la connotation civiliste de certains articles de droit comparé, il est important de noter la teinte franchement tricolore en colorant d'autres, portant eux uniquement sur le droit français. Ce point soulève un aspect méthodologique important: ce type d'écrits, spécialement tournés vers la France, va en principe comporter un grand nombre de références à la doctrine française. Si l'on reste à un niveau purement quantitatif, il est facile d'en tirer la conclusion d'une forte présence des auteurs français dans le paysage ; or, il nous semble que dans ce cas, nous sommes face à une surreprésentation assez artificielle. Est-il vraiment possible de tirer des conclusions quant à la diffusion de la pensée française dans une law review si les références ne sont finalement le fait que d'un ou deux juristes $?^{30}$ Encore une fois, l'analyse quantitative ne peut-être qu'un début et doit nécessairement être étayée par des éléments qualitatifs visant à éliminer ou expliquer ce type de distorsion. En l'espèce, Stumberg, qui écrit dans la Texas Law Review, n'est pas le seul auteur à se tourner spécifiquement vers la France ${ }^{31}$ et les nombreux articles sur ce pays présents dans la Michigan Law Review expliquent certainement le nombre élevé de références que l'on y trouve ${ }^{32}$. Là encore, une conclusion un peu rapide pourrait être tirée, celle d'un attrait particulier pour ce pays ; or, les facteurs contextuels, intellectuels ou individuels pouvant expliquer ce choix ne sont pas toujours faciles à mettre au jour. De même, d'un point de vue méthodologique, un tel attrait devrait être justifié par une étude en creux, c'est-à-dire en vérifiant que seule la France,

Instruments Laws of the Americas : a Comparative Study », Michigan Law Review, 43, 1944, p. 559-590; R. W. Millar, "The Premises of the Judgment as Res Judicata in Continental and Anglo-American Law », Ibidem, 39, 1940, p. 1-36.

${ }^{28}$ Voir sur ce point D. S. Clark, « The Modern Development of American Comparative Law : 1904-1945», American Journal of Comparative Law, 55, 2007, p. 587 ; R. Moore, « Le droit comparé aux États-Unis », Revue internationale de droit comparé, 46/3, 1994, p. 757-59 ; V. V. Palmer, «American Comparative Law : The Past One Hundred Years », Tulane Law Review, 75, 2000, p. 1093-1102.

${ }^{29}$ Tel nous semble être le cas pour la référence faite à Lyon-Caen et Renault, qui est d'ailleurs une référence indirecte : l'auteur dit la tirer de l'ouvrage Derecho Mercantil Mexicano de Tena. F. F. Barker et J. M. Cormack, «The Mercantile Act», art. cit., p. 224.

${ }^{30} \mathrm{C}$ 'est un aspect auquel nous avons été confrontées lors de recherches plus larges. Edwin Keedy, dans un article portant sur un point spécifique de la procédure pénale française, cite plus de soixante-dix juristes français. Or, cet article semble répondre à des fins uniquement pédagogiques et ne fait pas de lien entre les situations française et étatsunienne. De même, il ne semble pas être motivé par des considérations tenant à la procédure pénale américaine, mais plus par le fait, peut-être motivé par des facteurs purement personnels, que l'auteur a passé dix mois en France. "Preliminary Investigation of Crime in France - I, II et III ", University of Pennsylvania Law Review and American Law Register, 1939-1940, p. 385-424, p. 692-727 et p. 915-933. Même s'il n'a pas été publié dans l'une des quatre law reviews étudiés, cet exemple d'une possible distorsion des résultats nous semble intéressant.

${ }^{31}$ G. W. Stumberg, « French Legal History and Legal Historians », Texas Law Review, 6, 1928, p. $247-265$.

32 "Compte rendu du chapitre "Modern French Legal Philosophy" », Michigan Law Review, 20, 1921-1922, p. 464-466 ; R. B. Sobernheim, « Regulation of Labor Unions and Labor Disputes in France », Ibidem, 37, 1939, p. 1025-1077 ; J. P. Dawson, « The Codification of the French Customs », Ibid., 38, 1940, p. 765-800 ; A. Uhler, " The Doctrine of Administrative Trespass in French Law : An Analogue of Due Process », Ibid., 37, 1938, p. 209-235 ; M. Ploscowe, « The Investigation Magistrate (Juge d'Instruction) in European Criminal », Ibid., 33, 1935, p. 1010-1036. 
ou que majoritairement la France, est citée par la doctrine étatsunienne, et non d'autres pays. Or, un simple coup d'œil au sommaire de la Michigan Law Review montre que les auteurs se sont tour à tour intéressés à la Russie, au Luxembourg, à l'Écosse, à l'Allemagne ou à l'Italie ${ }^{33}$.

16. Dans les autres universités en revanche, les références sont le fait de nombreux auteurs différents : ils sont au nombre de vingt-et-un pour le Texas, vingt-deux pour la Californie du Sud et soixante pour la Californie, dans le cadre d'articles portant sur des sujets variés. Cela nous paraît aller dans le sens d'une relativement implantation de la doctrine française, meilleure en tout cas que si elle n'était le fait que d'auteurs isolés, spécifiquement tournés vers un point de droit français ${ }^{34}$. Cette diversité est un des points communs aux laws reviews étudiées, qui englobent également l'intérêt pour les matières semblables, comme vu plus haut. Toutefois, ces similitudes n'empêchent pas la mise au jour de spécificités locales.

\section{2. Des spécificités locales dans les états d'imprégnation civiliste}

17. En premier lieu, il est indispensable d'indiquer ce que nous entendons par imprégnation civiliste $»$ : ce terme peu sembler incongru appliqué au territoire américain, pourtant, certains auteurs n'hésitent pas à parler de "réception de la common law ${ }^{35}$, réception qui serait toujours en cours au milieu du XIX ${ }^{\mathrm{e}}$ siècle, sur diverses parties du continent. En effet, ce n'est que peu à peu que ce système s'est imposé aux États-Unis face aux systèmes juridiques des premiers colons venus de France ou d'Espagne, par exemple. Ainsi, les idées continentales furent reçues par le continent américain entre le $\mathrm{XIX}^{\mathrm{e}}$ siècle et le début du $\mathrm{XX}^{\mathrm{e}}$ siècle, « en nombre signifiant et dans des contextes différents $[\ldots] »^{36}$, emprunts délibérés ou infiltration semi-consciente ${ }^{37}$.

18. Le Texas est l'un de ces États ayant conservé des « éclats de droit civil dans son droit substantiel $»^{38}$ et où il existe des survivances d'institutions et de règles civilistes dans des champs variés, notamment en droit de la famille, concernant la protection du conjoint survivant $^{39}$. De façon identique, il a conservé les règles civilistes en matière de communauté de biens ${ }^{40}$, ce qui explique probablement le recours à la doctrine française dans un article

\footnotetext{
${ }^{33}$ E. Maxey, « Russian Raids on Neutral Commerce », Ibid., 3, 1904-1905, p. 1-8; T. P. Ion, « The International Status of the Grand Duchy of Luxemburg and the Kingdom of Belgium », Ibid., 13, 1914-1915, p. 368-390 ; R. W. Wyness, « Civil Pleading in Scotland », Ibid., 30, 1932, p. 545-581 ; J. P. Dawson, « Effects of Inflation on Private Contracts : Germany, 1914-1924», Ibid., vol. 33, 1934 ; P. B. Rava, « Italian Administrative Courts Under Fascism », Ibid., 40, 1942, p. 654-678.

${ }^{34}$ A noter, l'exception constituée par M. Radin, « Domicile in French Divorces », California Law Review, 13, 1924-1925, p. 513-514, que ne cite que quatre auteurs français.

35 C. S. Potts, «Early Criminal Law in Texas : From Civil Law to Common Law to Code », Texas Law Review, 21, 1942-1943, p. 394.

${ }^{36}$ M. Reinmann, «Introduction : Patterns of Reception », The Reception of Continental Ideas in the Common Law World, 1820-1920, Berlin, Dunker \& Humblot, 1993, p. 16.

${ }^{37}$ Ibidem.

${ }^{38}$ L. M. Friedman, A History of American Law, New York, Simon \& Schuster, 1973, p. 151.

${ }^{39}$ Il est possible de retracer « des influences espagnoles (et plus généralement, de droit civil et canon) sur le droit actuel du Texas ». H. W. Baade, « Reflections on the Reception (or Renaissance) of Civil Law in Texas », SMU Law Review, 55, 2002, p. 61.

${ }^{40}$ L. M. Friedman, A History of American Law, op. cit., p. 151.
} 
traitant de ce sujet ${ }^{41}$. Il semble en être de même pour certains points du droit des contrats, tels que la fraude ou l'erreur ${ }^{42}$.

18. La situation est similaire, voire peut-être amplifiée, en Californie puisqu'elle s'est dotée d'un Code civil en 1872. Cet État semble en outre faire un usage technique de la doctrine française pour tenter de régler, ou tout du moins d'éclaircir, un problème juridique. De nombreux articles font ainsi référence au «droit de l'eau ${ }^{43}$, ce qui n'est pas étonnant au vu du contexte : le partage des eaux du Colorado posent des difficultés depuis le XIX ${ }^{\mathrm{e}}$ siècle, non seulement entre les États-Unis et le Mexique, mais également au sein des États-Unis euxmêmes. La ruée vers l'or et le développement de l'industrie s'accompagne de l'augmentation des besoins hydrauliques, hors la Californie, dans la partie basse du fleuve, risque de se voir privée d'eau par les usages trop importants faits par les états en amont. Dès lors, la controverse fait rage : les états arides prônent la doctrine de l'appropriation antérieure, qui fonctionne sur la base du « premier arrivé, premier servi », tandis que les états côtiers, tels que la Californie, réclament l'application de la doctrine de la riveraineté ${ }^{44}$. Or, certains auteurs sont formels : ce principe, qui garantit à chaque riverain un droit égal sur l'eau, doit sa formulation au Code Napoléon, «et le droit européen moderne, particulièrement le droit français, a beaucoup à nous offrir par son exposé et son application $»^{45}$. Dans ce cas, il semble que l'utilisation de la doctrine française permette d'appuyer les arguments techniques allant dans le sens des intérêts californiens. À ce propos, nous pouvons constater que les revues californiennes n'utilisent pas des références identiques, ce qui peut indiquer des références orientées vers des buts institutionnels plutôt que locaux ou étatiques.

19. Quoi qu'il en soit, la doctrine française est présente dans de nombreux contextes. Nous pouvons la trouver dans des articles de droit comparé, mettant en jeu un cadre spécifiquement français ou européen, à propos de points techniques ou encore d'événements mondiaux ${ }^{46}$. Cette première observation, celle d'une utilisation plutôt diversifiée des auteurs français, doit être complétée par une autre modularité, celle visant les auteurs " de degré 4 », c'est-à-dire des auteurs qui sont reliés aux quatre universités étudiées.

\section{La représentation des auteurs de degré 4}

20. La visualisation sous forme de réseau n'est pas la représentation la plus adaptée ici, puisque nous savons déjà que chaque référence sera reliée aux quatre universités. Nous avons donc choisi de présenter les résultats obtenus sous forme de nuage de mots. Y figurent les noms des juristes français cités dans toutes les law reviews étudiées; plus la taille de la police est grande, plus le nom est mentionné. Duguit, Pothier, Pillet et Gény se trouvent donc être les

\footnotetext{
${ }^{41}$ J. E. Sebree, «Federal Taxation of Community Property », Texas Law Review, 12, 1933-1934, p. 398-414.

${ }^{42}$ B. Smith, "Correcting Mistakes of Law in Texas », Ibidem, 9, 1931, p. 309-332 ; J. W. Trade, "Benefits Obtained Under Illegal Transactions - Reasons For and Against Allowing Restitution », Ibid., 25, 1946-1947, p. $42-61$.

${ }^{43}$ Voir (tous nombres de références confondus) S. C. Wiel, « Origin and Comparative Development of the Law of Watercourses in the Common Law and in the Civil Law », California Law Review, 6, 1917-1918, p. $245-267$; J. C. S., " Comment on Recent Cases : Water Rights - Surface Waters - Similarity of Common Law and Civil Law », Ibidem, 8, 1919-1920, p. 199-201 ; L. A. C., «Comment on Cases : Waters - Flood Water Doctrine in California », Ibid., 10, 1921-1922, p. 90-91; S. C. Wiel, «Political Water Rights», Ibid., p. 111-119; S. C. Wiel, « The Pending Water Amendment to the California Constitution and Possible Legislation », Ibid., 16, 1928, p. 169-207.

${ }^{44}$ W. Stegner, Lettres pour un monde sauvage, Paris, Gallmeister, 2015, p. 152.

${ }^{45}$ S. C. Wiel, " Origin and Comparative Development of the Law of Watercourses in the Common Law and in the Civil Law » art. cit., p. 245.

${ }^{46} \mathrm{Il}$ y a une forte présence des auteurs français dans des textes à propos de la Première Guerre mondiale.
} 
auteurs les plus présents. La réception, ou tout du moins la lecture de ce dernier ayant déjà fait l'objet de plusieurs études ${ }^{47}$, nous nous concentrerons sur d'autres auteurs, à savoir Duguit, Hauriou et Esmein, Pothier et Domat, pour terminer avec Pillet.

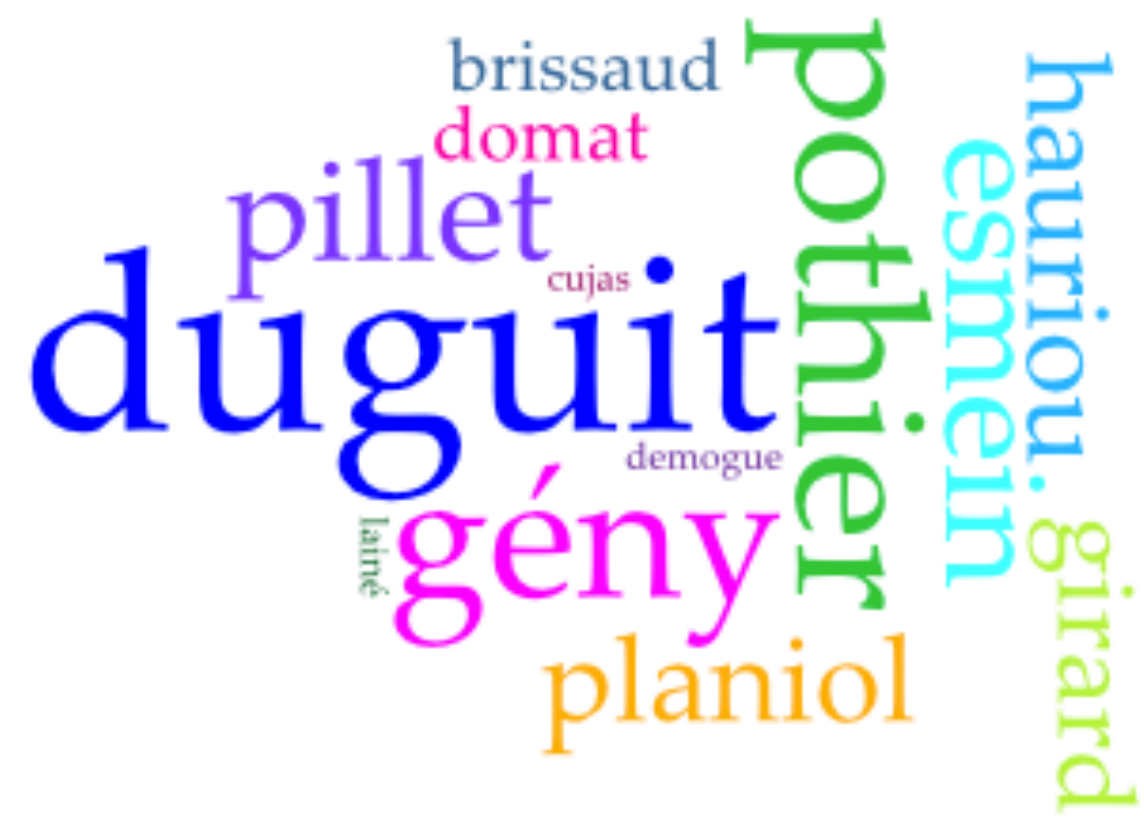

Représentation graphique des auteurs de doctrine française cités par les quatre universités étudiées

\section{Léon Duguit}

21. Comme le montre le nuage de mots, Duguit est l'auteur français le plus mentionné dans notre réseau, ce qui n'a rien d'étonnant, puisque c'est le juriste français le plus cité aux ÉtatsUnis, toutes law reviews confondues, entre 1870 et $1940^{48}$. Plusieurs raisons expliquent cette situation : nombreux de ces ouvrages importants ont été partiellement traduits en anglais et publiés dans des revues importantes ${ }^{49}$; de plus, Duguit a visité les États-Unis et a donné une série de conférences à l'université Columbia ${ }^{50}$. Au vu de ces canaux de diffusion (invitations,

\footnotetext{
${ }^{47}$ M. Franklin, «L'influence de M. Gény sur les conceptions et les méthodes juridiques aux États-Unis », Recueil d'études en l'honneur de François Gény, Paris, Recueil Sirey, 2, Paris, 1934 ; A. Tate, «Book Review, Method of Interpretation and Sources of Private Positive Law by François Gény », Louisiana Law Review, 27, 1965, p. 577 ; J. Mayda, François Gény and Modern Jurisprudence, Baton Rouge, Louisiana State University Press, 1978; D. Kennedy et M.-C. Belleau, «François Gény aux Etats-Unis », dans C. Thomasset, J. Vanderlinden et Ph. Jestaz (dir.), François Gény, mythes et réalités, Dalloz, Bruylant et éd. Y. Blais, Bruxelles, 2000, p. 295-320 ; T. Kirat, « Les théories de la jurisprudence de François Gény et Édouard Lambert et la common law: remarques sur la position de la case-law method par rapport à l'œuvre des deux juristes français », Ibidem, p. 321-341 ; W. A. Penfold, «An Ineluctable Minimum of Natural Law. François Gény, Oliver Wendell Holmes, and the limits of Legal Skepticism », History of European Ideas, 37, 2011, p. 37 ; F.X. Licari, «François Gény en Louisiane», dans O. Cachard, F.-X. Licari et F. Lormant (dir.), La pensée de François Gény, Paris, Dalloz, 2013.

${ }^{48}$ Que l'absence de référence nous soit pardonnée, ces résultats provenant de nos recherches en cours.

${ }^{49}$ Par exemple, «The Law and the State », Harvard Law Review, 31, 1917-1918, pp. 1-180 ; «Collective Acts as Distinguished from Contracts », Yale Law Journal, 27, 1917-1918, p. 753 ou « Objective Law », Columbia Law Review , 20, 1920, p. 817.

${ }^{50}$ L. Duguit, Souveraineté et liberté - Leçons faites à l'Université Columbia (New-York) 1920-1921, Paris, Félix Alcan, 1922.
} 
traductions...), cette circulation semble s'inscrire dans un modèle fondé sur une communauté d'interconnaissance, communauté qui se constitue grâce à des visites ou des échanges épistolaires et retraçable grâce à l'intertextualité qu'elle génère, telle que les références ou les traductions ${ }^{51}$.

22. Traditionnellement, on imagine que ce type de communauté a pour base l'admiration mutuelle entre ses acteurs, ou au moins unilatérale. La situation paraît ici assez différente. Il est vrai qu'à l'origine Laski, le traducteur de Duguit aux États-Unis, le considérait comme le publiciste le plus important de son temps ${ }^{52}$, Angleterre et États-Unis compris. Pourtant, sa rencontre avec Duguit ne semble pas avoir été un succès : "Laski écrivit à Holmes que Duguit était un "diamant brut avec de bonnes idées, mais aussi pour une grande part ce que Frankfurter appellerait un faussaire. Il cite de l'allemand dans ses livres, mais j'ai découvert qu'il ne le lisait pas, et il a ce patriotisme criard qui fait de la rhétorique un substitut à l'argument" 53 ». Dès lors, les références à Duguit seraient suscitées plus par un intérêt d'estime que par un réel engouement ${ }^{54} \ldots$ voire peut-être par une opposition à ses idées : par exemple, ni Holmes ni Pound ne sont convaincus par ses théories ${ }^{55}$. En outre, il est vrai que Duguit a été publié dans de prestigieuses revues, telle que la Harvard Law Review : toutefois, concernant cette dernière, l'article de Duguit doit être compris comme appartenant à une série d'articles d'auteurs étrangers, destinés à combler le vide laissés par les contributeurs étasuniens partis à la guerre ${ }^{56}$.

23. Ainsi, la présence importante de mentions de Duguit n'emporte pas forcément l'adhésion ou la réception de ces idées, ce qui incite à la prudence dans l'interprétation de ce genre de résultats. Mais quand bien même la totalité de ces références ne seraient faites que dans le but d'éreinter la pensée de Duguit, elles prouvent que ce dernier est largement lu, comme on peut le constater sur le diagramme suivant, où le point central représente Duguit. En plus d'être lu, sa pensée nous semble également largement connue : s'il peut être évoqué en note de bas de page dans le cadre de points de droit et parmi d'autres auteurs, son nom apparaît le plus souvent en corps de texte, au côté par exemple d'autres références de la pensée européenne, tels que Stammler ou Kelsen ${ }^{57}$. Un auteur, dans les lignes introductrices de sa recension d'ouvrage, nous dit en parlant de la démarche de l'auteur que «d'Ulpien à Duguit, c'est un long chemin à parcourir en pensée juridique comme en histoire du droit $\rangle^{58}$ et un autre corrige un de ses collègues, estimant que dans son ouvrage, « la position de M. Duguit sur la question de l'État est quelque peu mal comprise ${ }^{59}$.

\footnotetext{
51 P.-Y. Saunier, «Les régimes circulatoires du domaine social 1800-1940: projets et ingénierie de la convergence et de la différence », Genèses, 71, 2008/2, p. 16.

52 C. Harlow, "The Influence of Léon Duguit on Anglo-American Legal Thought», dans Autour de Léon Duguit, Bruylant, Bruxelles, 2011, p. 236.

53 Ibidem.

${ }^{54}$ J.-M. Blanquer et M. Milet, L’invention de l'État : Léon Duguit, Maurice Hauriou et la naissance du droit public moderne, Paris, Odile Jacob, 2015, p. 117.

${ }_{55}$ C. Harlow, «The Influence of Léon Duguit on Anglo-American Legal Thought », op. cit., p. 238.

${ }^{56}$ Ibidem, p. 235.

${ }^{57}$ H. Rottschaefer, « Corporate Personality - F. Hallis », Michigan Law Review, 29, 1930-1931, p. 536.

${ }^{58}$ V. H. Fowler, " The Revival of Natural Law Concepts - C. G. Haines », Ibidem, p. 964.

${ }^{59}$ M. Radin, « The Origin of the State - R. H. Lowie », Southern California Law Review, 2, 1928-1929, p. 96.
} 


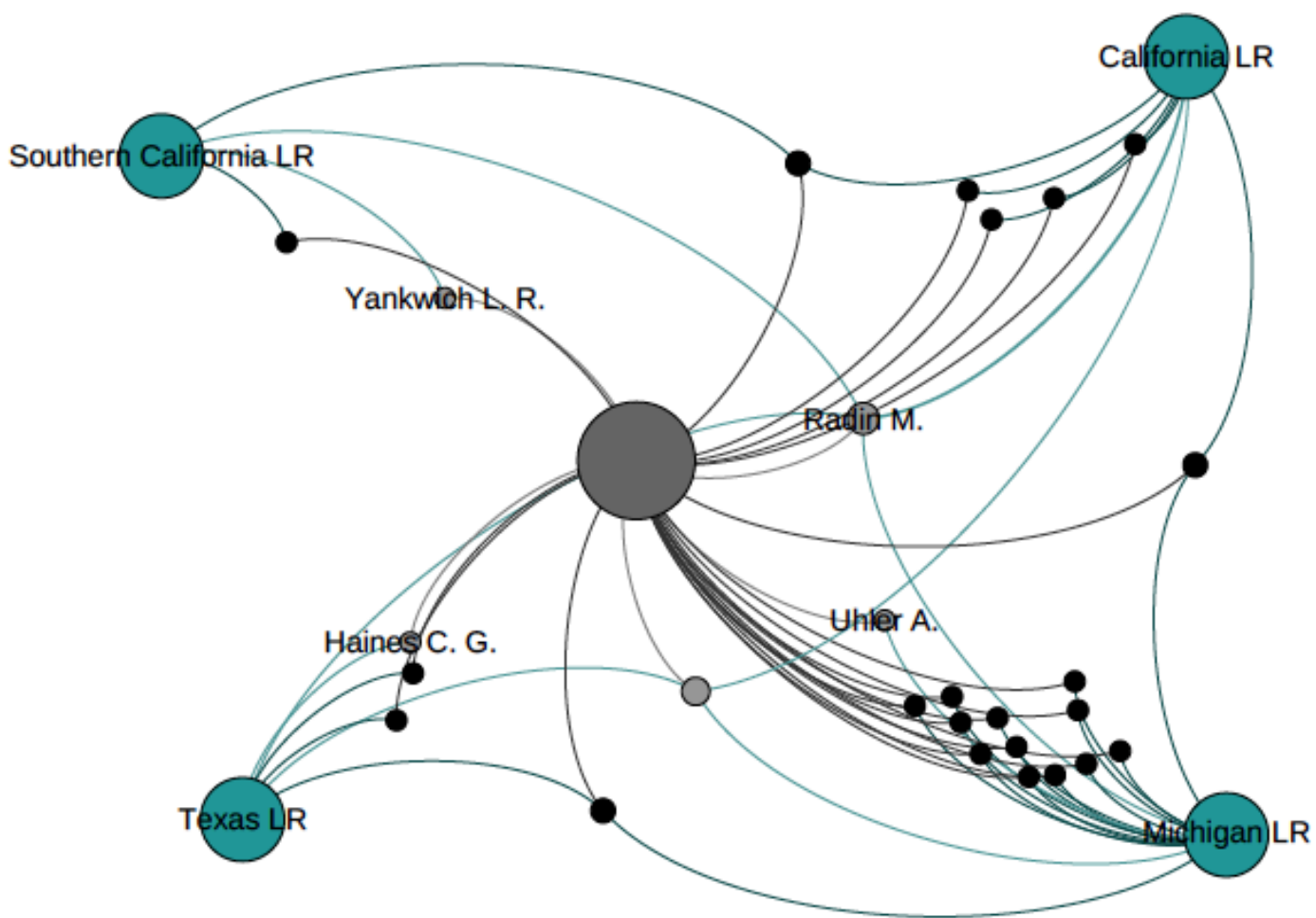

Représentation des références faites à Duguit au sein des law reviews

24. Cette forte présence explique peut-être celle des deux autres noms que nous allons désormais étudier, ceux de Hauriou et d'Esmein.

\section{Maurice Hauriou et Adhémar Esmein}

25. Tous deux figurent en bonne place dans le nuage de mots représentant les auteurs les plus cités ; ils sont publicistes, comme Duguit, mais n'ont jamais fait de séjours aux États-Unis et seul un texte de Hauriou a été traduit en anglais et publié dans la Harvard Law Review ${ }^{60}$. Malgré ces différences, ils se trouvent tous deux en bonne place dans le nuage de mots représentant les auteurs les plus cités. Dès lors, quelles hypothèses peut-on formuler pour expliquer cette situation?

26. La première peut se rapporter à leur champ de spécialité ; en effet, il aurait été possible d'imaginer qu'ils traitent de thématiques susceptibles d'intéresser les États-Unis à l'époque où ce pays connaît un développement de l'administration, ou simplement dans le cadre plus large d'interrogations autour de la séparation des pouvoirs. Pourtant, le droit administratif ou le droit constitutionnel n'occupent qu'une très faible place dans le panorama des références ${ }^{61}$, comme le montre le diagramme ci-dessous.

\footnotetext{
${ }^{60}$ M. Hauriou, «Interpretation of the Principles of Public Law », Harvard Law Review, 31, 1917-1918, p. 813.

${ }^{61} 5,54 \%$ des références ont trait au droit administratif et $2,17 \%$ au droit constitutionnel, contre près de $26 \%$ pour le droit international.
} 


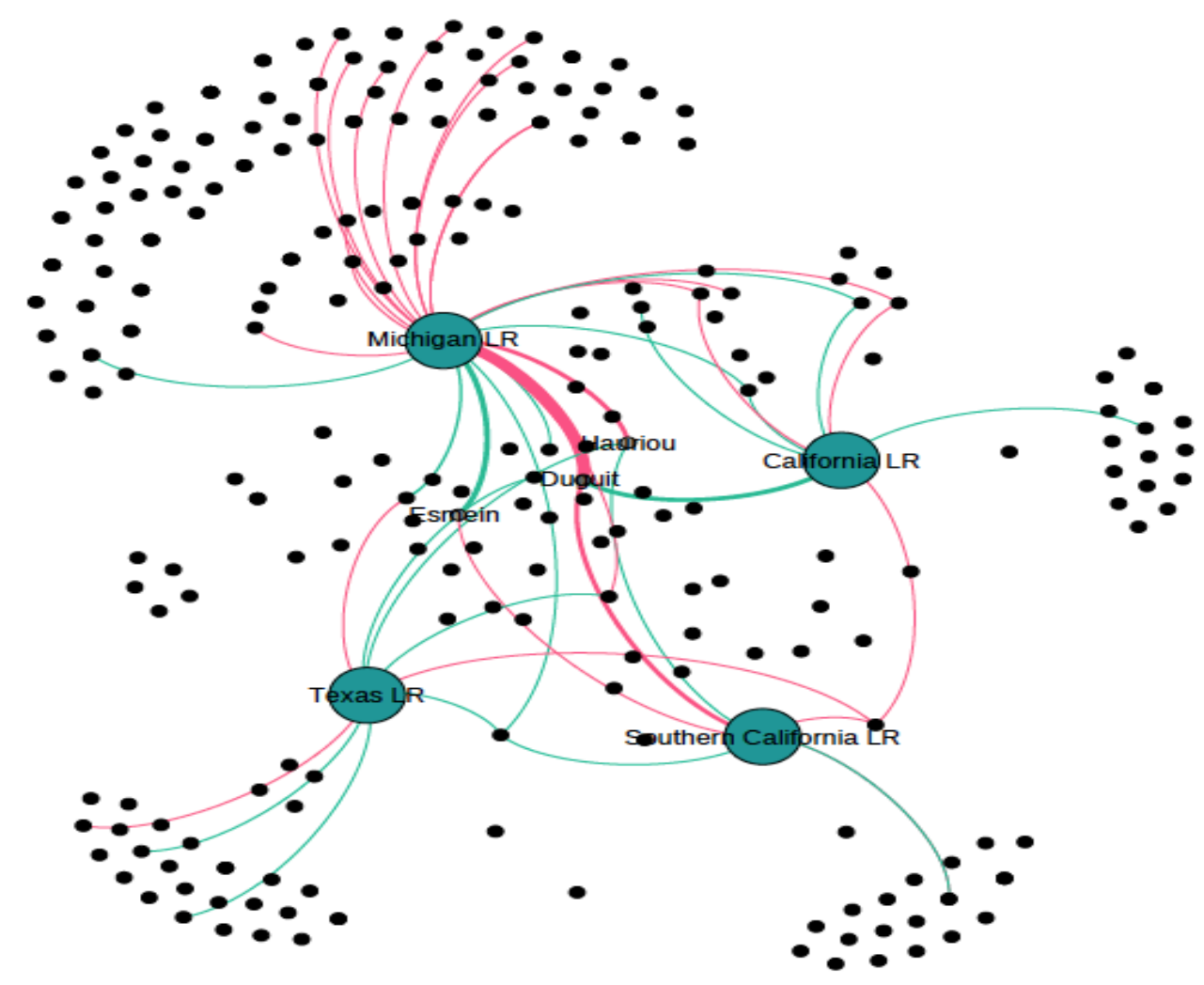

Représentation de la répartition des citations entre les articles consacrés au droit constitutionnel (en vert) et ceux dédiés au droit administratif (en rose).

27. Une autre explication peut être fournie en franchissant un nouveau cap dans l'analyse de réseaux. Dans le paragraphe sur Duguit, nous sommes restées finalement au stade de la dyade, c'est-à-dire à la relation entre deux personnes : Duguit est cité par X, ou par Y, ou par Z. Ici, il nous semble qu'analyser la présence d'Hauriou (ou d'Esmein) suppose de passer au stade de la triade, particulièrement intéressante dans ce cadre. Chez certains pionniers de la méthode structurale, la triade, donc la relation entre trois personnes, est la structure élémentaire pour l'émergence des formes sociales : la société ne naît pas avec deux mais avec trois individus, car c'est à cette échelle qu'émerge l'entité supra-individuelle qu'est le groupe ${ }^{62}$. Il nous semble effectivement que des auteurs tels qu'Hauriou ou Esmein s'inscrivent dans ce type de structure, surtout si l'on s'intéresse à la formation de ces triades. Mettons ainsi qu'A et B aient des liens très forts, tels que Pound et Duguit; et que A et C aient également des liens très forts, tels que Duguit et Hauriou. Selon certains, il est plus que probable qu'une relation entre $\mathrm{B}$ et $\mathrm{C}$, donc entre Pound et Hauriou, finissent par se former ; certains vont même jusqu'à considérer qu'une triade formée entre $\mathrm{A}-\mathrm{C}$ et $\mathrm{A}-\mathrm{B}$ sans lien entre B-C est une triade « interdite $»$, donc impossible ${ }^{63}$.

28. Ainsi, pour conclure, nous avons vu qu'il existe des liens assez forts entre Duguit et la doctrine américaine; il existe également des liens très forts entre Duguit et Hauriou et Esmein, tous deux étant abondamment cités dans ses ouvrages. Or, il nous paraît raisonnable, voire de «bonne pratique scientifique» que les universitaires étatsuniens interpellés par

\footnotetext{
${ }^{62}$ J. Brailly, F. Eloire et G. Favre et al., «Explorer les réseaux à l'échelle de la triade : l'apport des modèles statistiques ERGM », L'Année sociologique, 67, 2017/1, p. 219-254.

${ }^{63}$ M. Granovetter, «The Strenght of Weak Ties », American Journal of Sociology, 78, 1973/6, p. 1360-1380.
} 
Duguit aient fini par s'intéresser aux auteurs gravitant étroitement autour de lui. C'est peutêtre la même théorie qui explique la présence de deux autres auteurs que l'on peut associer, Pothier et Domat, mais il nous semble là devoir aller chercher l'explication ailleurs.

\section{Robert-Joseph Pothier et Jean Domat}

29. La présence de ces deux civilistes de l'Ancien Régime dans le panorama américain ne peut que soulever des questions, et deux en particulier: pourquoi des auteurs français si anciens sont cités aux États-Unis ? Pourquoi des auteurs civilistes sont cités aux États-Unis? En réalité, il exista une vraie francophilie et une véritable admiration pour la France après la Guerre d'indépendance, en raison de l'hostilité envers l'ennemi anglais et de l'admiration suscitée par le Code Napoléon ${ }^{64}$. Ainsi, avant le milieu du XIX ${ }^{\mathrm{e}}$ siècle, les idées continentales ont eu une plus grande influence aux États-Unis qu'en Grande-Bretagne; le prestige du droit romain, la solution à de nombreuses questions encore en suspens en droit anglais, l'élégance des codes français étaient pour beaucoup dans cette attraction ${ }^{65}$. Dès lors, " des livres de droit français, Domat et Pothier entre autres, eurent vite fait de traverser l'Atlantique - le Traité des Obligations de Pothier fut traduit et publié en 1802 en Caroline du Nord ${ }^{66}$. L'examen des décisions de justice montre que les civilistes français, au début du XIX $\mathrm{X}^{\mathrm{e}}$ siècle, étaient très fréquemment cités par les juges et les avocats, particulièrement dans trois cas : 1) quand la common law était inexistante, obscure ou erronée, 2) quand il s'agissait des principes fondamentaux de justice, 3) quand, et c'était le cas le plus fréquent, le droit étranger était identique au droit américain ${ }^{67}$.

30. Néanmoins, notre étude se déroule plus d'un siècle après, et les mêmes noms sont toujours retrouvés. Le sont-ils pour les mêmes raisons qu'auparavant? Nous le pensons... à condition de mettre au jour certaines de ces véritables raisons. Par exemple, un des géants du droit anglais, le juge Kent, truffait ses écrits de références au code civil, à Pothier et à la doctrine civiliste en général. Pour autant, il a été démontré qu'il n'utilisa pas vraiment les idées civilistes pour développer le droit américain ; de plus, il aurait souvent été plus simple pour lui de se référer à un mécanisme existant en common law plutôt que d'user de citations continentales, qu'il comprenait parfois mal ${ }^{68}$. Nous sommes face ici à ce qui a pu être appelé des citations de « façade », ayant pour pendant juridique " l'argument d'autorité ». La citation d'autorités étrangères a pu impressionner les collègues contemporains de Kent; se rajoute à l'époque qui nous intéresse une dimension devenue historique, ce qui peut rajouter encore au poids symbolique de la citation, même dénuée d'effet pratique. Face à ces références « pour la galerie », le dernier auteur examiné, Pillet, semble lui correspondre à la fonction tout à fait inverse.

\footnotetext{
${ }^{64}$ D. Seipp, « Our Law, Their Law, History and the Citation of Foreign Law », Boston University Law Review, 86, 2006, p. 1425.

${ }^{65}$ M. Reinmann, "Introduction: Patterns of Reception», dans The Reception of Continental Ideas in the Common Law World, 1820-1920, Berlin, Dunker \& Humblot, 1993, p. 9.

${ }^{66} \mathrm{C}$. Kirby-Légier, « Droit anglais et droit américain : divorce de raison? », dans Langue et culture : mariage de raison ?, R. Greenstein (dir.), Paris, Publications de la Sorbonne, 2009, p. 129.

${ }^{67}$ R. H. Helmholz, « Use of the Civil Law in Post-Revolutionary American Jurisprudence », Tulane Law Review, 66, 1991-1992, p. 1679.

${ }^{68}$ M. Reinmann, «Introduction : Patterns of Reception », op. cit., p. 10.
} 


\section{Antoine Pillet}

31. Ici, la forte présence de l'auteur parait en adéquation avec la mobilisation de sa matière, qui, on le voit, irrigue de façon assez régulière et abondante les références des quatre universités, et représente plus d'un quart des citations totales.

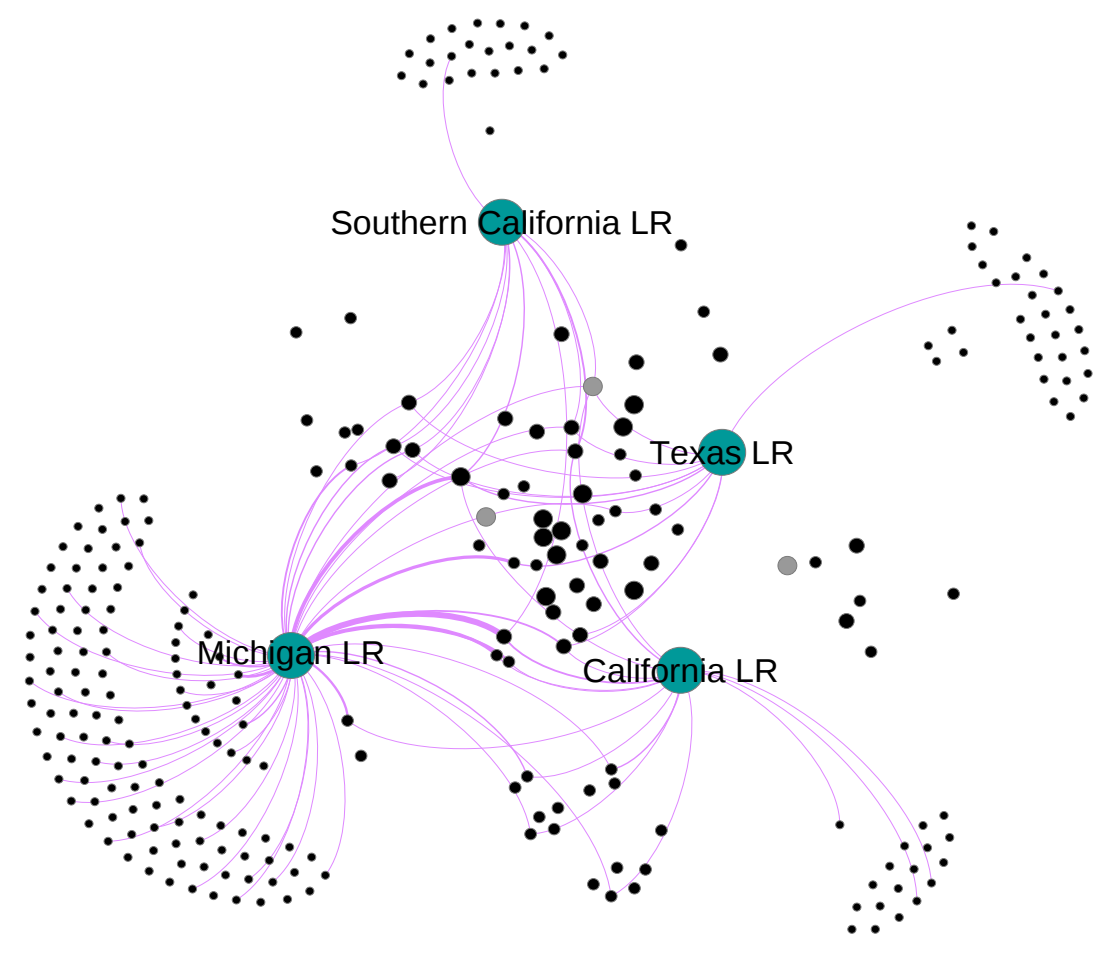

Représentation des citations ayant trait au droit international

32. Il n'y a rien d'étonnant à cette mobilisation, qui nous paraît rentrer dans un cadre pragmatique, voire utilitariste assez classique : le droit international, dit " Conflict of Laws » en Amérique du Nord, est né depuis quelques siècles mais est plus que jamais sollicité par la modernisation des communications, des échanges et le basculement des rapports de force après la Première Guerre mondiale. Des conflits de loi se posent à propos des thèmes les plus variés (divorce, commerce, droit aérien) et avec de plus en plus de pays. Face à ces problèmes nouveaux, les auteurs se tournent vers la doctrine compétente, parmi qui, en l'espèce, se trouve Pillet, considéré par beaucoup comme le fondateur du droit privé. Nous sommes cette fois face à des références motivées par la compétence de celui à qui l'on se réfère.

\section{Conclusion}

33. Au terme de cet exposé, la première constatation qui peut être faite est celle de l'hétérogénéité. La présence de références à la doctrine française est réelle et quasiment quantifiable, mais ces données ne doivent pas faire oublier l'essentiel : au delà du souhait vertueux d'une communauté d'idée et d'une sociabilité scientifique, les références sont orientées et répondent à des buts. A travers les exemples exposés ici, la typologie suivante peut être proposée :

- la référence individualisée: elle se voit dotée d'une sorte d'intuitu personae et l'auteur est cité « parce que c'est lui ». On admire ses idées ou on les conspue, on peut l'avoir rencontré personnellement ou échanger avec lui une correspondance... Le 
paradoxe étant que cet intuitu personae n'est en aucun signe de réception ou d'assimilation de ces idées sur le territoire étranger. Ce type de référence, illustrée par le cas de Duguit, nous paraît créer un dialogue, parfois direct, par notes de bas de page interposées, entre le citant et le cité.

- la référence par connectivité : accompagnant généralement les références individualisées, elle nous parait caractériser particulièrement l'activité de recherche. En effet, il s'agit ici, après avoir étudié la pensée d'un auteur, d'aller plus loin et de s'intéresser aux auteurs référencés par cet auteur, créant ainsi de nouveaux liens, comme ce fut peut-être le cas pour les auteurs de droit public gravitant autour de Duguit. Cela peut initier un dialogue entre le citant et le cité, mais ce dernier peut aussi rester ignorant de ce lien.

- la référence d'autorité : elle peut, elle aussi, être parfois caractéristique de l'activité scientifique... Cette référence appelle un auteur, ancien ou contemporain, éminemment connu dans son champ et n'a parfois pas d'utilité pratique dans le contexte, ni même de réel rapport avec le champ traité. Il nous semble que ce faisant, le citant crée un lien avec lui-même, ou tout du moins avec son image sociale. Par le biais de la référence, il montre son érudition et son capital culturel.

- la référence d'utilité : cette dernière est motivée par des considérations pratiques. Le cité, en général une sommité de son domaine, est convoqué à bon escient à l'appui de démonstrations pratiques ou en vue de trouver des solutions à un problème juridique. Celle-ci nous paraît la plus destinée au lecteur scientifique, voire au praticien : utile pour le citant, elle l'est également pour le lecteur désireux de vérifier les hypothèses ou de confronter les opinions.

Cette typologie n'est bien sûr en aucun cas exhaustive, ni exclusive.

34. Pour terminer, il reste un point à aborder, laissé en suspens : le nombre de références françaises au sein de l'université du Michigan. Certes, les premiers colons ayant exploré le territoire étaient français, mais cela remonte au XVII ${ }^{\mathrm{e}}$ siècle ; les territoires seront eux cédés à l'Angleterre en 1763. Cela peut-il suffire à expliquer cette francophilie ? L'hypothèse prise seule semble ténue. Une autre hypothèse est celle d'une law review plus ancienne que les autres $^{69}$, donc mieux implantée dans le paysage juridique et plus susceptible d'attirer des plumes diversifiées, aux centres d'intérêts plus larges. Malgré tout, et de manière plus générale, peut-on toujours expliquer le tour pris par un réseau d'idées ? En l'occurrence, il nous revient en mémoire cet entrefilet trouvé au hasard de la revue Enquêtes et documents de l'enseignement supérieur : une bibliothèque universitaire américaine ayant été ravagée par un incendie, il était demandé à chaque faculté française d'expédier les doublons de ses ouvrages afin de regarnir ses étagères. Il est tentant d'imaginer les conséquences de ces envois et la création potentielle de nouvelles communautés d'idées. Il est encore plus tentant de rappeler que les circulations des idées sont émaillées du même aléa que celui dominant toute relation sociale.

\footnotetext{
${ }^{69}$ Le premier numéro date de 1902.
} 